\title{
Polycyclic Aromatic Hydrocarbons in Different Varieties of Rice (Oryza sativa) from Yobe State, Nigeria
}

\author{
Joseph Clement Akan ${ }^{1}$, Joshua Yohanna Dawa ${ }^{1}$, Lawan Inuwa Bukar ${ }^{1} \&$ Zakari Muhammed $^{1}$ \\ ${ }^{1}$ Department of Chemistry, University of Maiduguri, Maiduguri, Nigeria \\ Correspondence: Joseph Clement Akan, Department of Chemistry, University of Maiduguri, P.M.B 1069, \\ Maiduguri, Borno State, Nigeria. Tel: 234-80-3600-0506. E-mail: joechemakan@yahoo.com
}

Received: August 10, 2018

Accepted: September 11, $2018 \quad$ Online Published: September 24, 2018

doi:10.5539/ep.v7n2p21

URL: https://doi.org/10.5539/ep.v7n2p21

\begin{abstract}
The present study determined the levels of sixteen polycyclic aromatic hydrocarbons (PAHs) in rice (Oryza Sativa) samples from six agricultural locations in Bade and Karasuwa Local Government Areas, Yobe State, Nigeria. Four varieties of rice (FARO 42, 44, 45 and 52) were collected for this study. The concentrations of PAHs in the study rice samples were lower than the maximum allowable concentration (MAC), average daily dose (ADD) of PAHs in the different variety of rice from the six agricultural locations shows that FARO 44 had the highest ADD $\left(5.84 \times 10^{11} \mathrm{mg} / \mathrm{kg}\right)$, while FARO 52 shows the lowest ADD $\left(1.20 \times 10^{15} \mathrm{mg} / \mathrm{kg}\right)$. The potential for non-carcinogenic PAHs in this study revealed that FARO 42 has the highest hazard index $\left(2.04 \mathrm{x} 10^{11} \mathrm{mg} / \mathrm{kg}\right)$. Result from incremental lifetime expectancy cancer risk shows that FARO 44 from Jawa had the highest value $\left(4.19 \times 10^{10} \mathrm{mg} / \mathrm{kg}\right)$, while the lowest value $\left(7.61 \times 10^{15} \mathrm{mg} / \mathrm{kg}\right)$ was recorded for FARO 52 from Rina Kuna agricultural location. Results from the present study shows that the rice samples from the study locations are safe for human consumption and not significantly contaminated by PAHs.
\end{abstract}

Keywords: rice, FARO, PAHs, ADD, risk assessment

\section{Introduction}

Polycyclic aromatic hydrocarbons (PAHs) are aromatic hydrocarbons with two or more fused benzene rings in various structural configurations (Masih and Lal, 2014; Sharma 2014). The present of PAHs has been reported in many food items including edible oils (Hao et al., 2015), meat (Li et al., 2015), fish (Nwaichi and Ntorgbo, 2016), (Akan et al., 2017), (Zakari et al., 2017). Research study on mutagenic and/or carcinogenic PAHs have also been found in cooked rice (Essumang, 2014). The consumption of different types of food my human is an important pathway for human exposure to PAHs, and dietary intake is one of the exposure rout to PAHs from (Shen et al., 2014). In the past years, there had been increased in the consumption of rice in Nigeria, and imported and locally rice are widely consumed as the most stable food. There have been increased in the consumption of rice on a daily basis to approximately $80 \%$. According to the Rice Council of America, two-thirds of the people in the world consider rice a staple food. High rate of industrialization is gradually leading to contamination and deterioration of the environment and pollution is likely to reach alarming levels in the environment. PAHs are found in various ecosystems and are pollutants of important concern as a result of their potential toxicity, mutagenicity and carcinogenicity (Klein, 2014).

Rice (Oryza sativa) is one of the most cultivated crops in the world. It is the third most important cereal grown and consumed globally after wheat and maize. It belongs to the family Poaceae. Rice is widely grown and is among the cereals crop of importance in the world production. The crop was previously assumed to be principally Asiatic; it is now grown in large quantity in many parts of the world including Africa, Asia, Australia and South America (FAO, 2006). It constitutes the staple food for 2.5 billion people (Ito and Shikawa, 2004) and rice growing is the largest single use of land for producing food, covering $9 \%$ of the earth's arable land. In poor countries of Asia, rice account for about $50-80 \%$ of daily caloric in-take. In Africa, rice serves as an important food crop with a yield of about $5082 \mathrm{~kg} \mathrm{ha}^{-1}$ (FAO, 1972). Rice straw serves as feed for livestock and also serves as raw material for many industrial products. It is used in manufacturing of beers, wines and spirits in Japan and China. Bran oil is used in making insecticides, anti-corrosive and rust-resistance oil. Wax can also be obtained from the bran (Adebayo and Salawu, 2010).

In Nigeria, rice is cultivated in almost all ecological belts available in the country as they all provide favourable 
environments to support the crop. This has made rice a staple crop in the nation as it is consumed by almost every individual in the nation in different forms. FARO is the acronym for Federal Agricultural Research Oryza in Nigeria. Faro 40, 41, 42 and 43 are medium-duration varieties recommended for most environments. FARO 40, a composite developed in Nigeria, is resistant to lodging and diseases. Bade and Karasuwa Local Government Areas are located in Yobe State (Map 1), intense rice activities take place in the area, and is $10 \mathrm{~km}$ along the Lake Chad Basin area. The people in the areas are $98 \%$ predominantly farmers out of this, merely $65 \%$ are commercial rice cultivating farmers; and the cultivated rice are sold to other parts of Nigeria. Faro 44 and Faro 52 appear to be the most widely grown variety in Gashua by farmers, since they are common to all sites visited during the field survey. The wetlands used for the cultivation of rice within the study areas received water from three main rivers in the Hadejia- Jamaare - Yobe River System and the rivers Hadejia, Jarnaare and Komadugu - Yobe. The Hadejia River is formed by the confluence of river Kano and Challawa at Tamburawa. The rivers carries chemical pollutants from the highly industrial city of Kano and Jos to the wetland which is used for the cultivation of rice. Studies conducted out by Akan et al. (2017), Zakari et al. (2017) in water, sediment and fish samples from the study area consistently showed alarming levels of PAHs. However, PAHs contamination of rice cultivated within the wetland has not been taken into consideration and had been ignored generally in the past years. Hence, the objectives of this study are to determine the levels of PAHs in varieties of rice and to evaluate the level of health risk associated with PAHs exposure through consumption of rice.

\section{Materials and Methods}

Rice samples (Faro 42, 44, 45 and 52) were collected from Mashayan Bululu, Jawa and Rina Kunu in Bade Local Government Area and Wachakal, JajiMaji and Wachakal Ngurodi in Karasuwa Local Government Area, Yobe State, Nigeria (Map 1). The different varieties of rice samples were transported to the laboratory and stored at $25^{\circ} \mathrm{C}$. Analysis for PAHs in rice samples were carried out in accordance with USEPA (2000) 8082 analytical method. The extract was thereafter analyzed using Agilent 7890A GC/MS previously calibrated with PAHs standards. The estimation of human dietary Dose PAHs dosed (mg kg- $\left.{ }^{1}-^{1}\right)$ occurring over a lifetime were determined. For this study, benzo(a)pyrene $(\mathrm{BaP})$ equivalent dose of mixture of carcinogenic PAH compounds were calculated for carcinogenicity using the following equation adopted by World Bank, (2014).

$$
\mathrm{ADD}=\frac{\mathrm{TEQ} \times \mathrm{IR} \times \mathrm{CF}}{\mathrm{BW}}
$$

These exposure assumptions were made to be consistent with EPA guidance on assumption on reasonable maximum exposure (USEPA, 1999). Where IR is the ingestion or intake rate of carcinogenic PAHs based on average fish consumption rate set at $68.5 \mathrm{~g}$ day- ${ }^{1}$ per person from the annual per capital fish consumption of 25 $\mathrm{kg}$ for Nigeria (FAO, 2008) CF is the conversion factor $\left(0.000001 \mathrm{mg}_{\mathrm{gg}}{ }^{1}\right)$ and average body weights (BW) in Nigeria is estimated to be $70 \mathrm{~kg}$ (Benson et al., 2016a, b). 


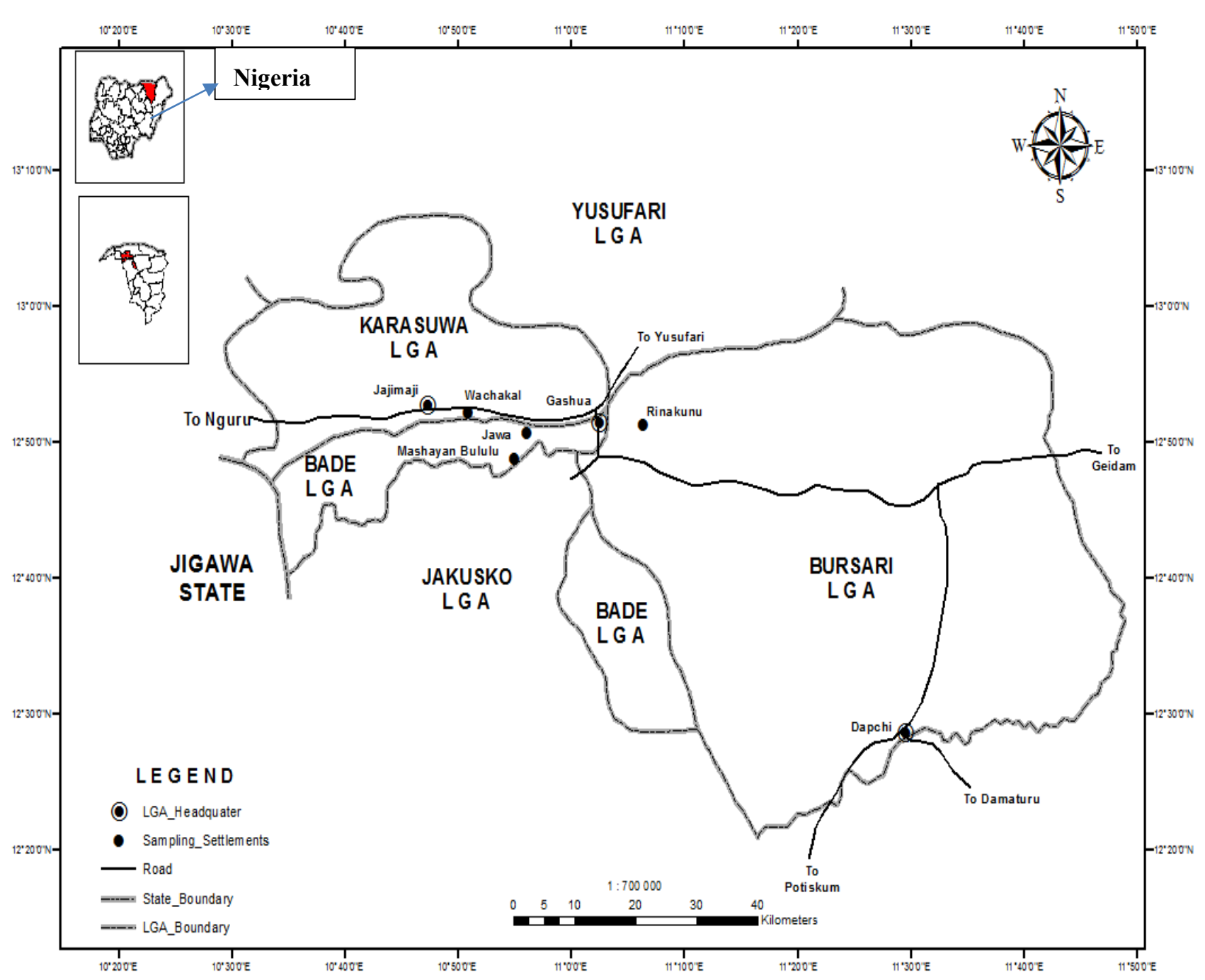

Source : Department of Geography, Univers ity of Maiduguri (2018).

Figure 1. Map of study areas showing sampling locations

Risk associated with dietary exposure to non-carcinogenic PAHs was evaluated using hazard quotients approach. Hazard quotients represent a ratio of the exposure dose for each PAH divided by an oral chronic reference dose (RfD). Hazard quotient (HQ) = Average daily dose (ADD)/RfD. The total risk due to exposure to mixture of carcinogenic PAHs is the product of the dietary carcinogen exposure dose (mg kg- ${ }^{1} \mathrm{BW}^{\mathrm{d}-{ }^{1}}$ ) and benzo(a)pyrene's slope factor value.

Risk (carcinogenic) $=$ Average daily dose $\mathrm{x}$ slope factor

\section{Results}

The mean concentrations of some polycyclic aromatic hydrocarbons in rice samples from different location of Bade Local Government Area, Yobe State, Nigeria are presented in Table 1. Since all the sample locations in the current study contained PAHs, it clearly demonstrates the widespread nature of these persistent compounds. The total PAH contents from all the sampling locations reveals that FARO 42 and 44 showed higher values of 6.68 $\times 10^{5}$ and $5.60 \times 10^{5} \mathrm{mg} / \mathrm{kg}$ respectively from Jawa agricultural location, whereas Mashayan Bululu and Rina Kunu locations showed relatively lower values of $1.44 \times 10^{8}$ and $1.72 \times 10^{8} \mathrm{mg} / \mathrm{kg}$ respectively. Table 2 present the mean concentrations of some polycyclic aromatic hydrocarbons in rice samples from Karusawa Local Government Area, Yobe State, Nigeria. Faro 52 from Wachakal location recorded the highest total concentration 
of $2.50 \times 10^{8} \mathrm{mg} / \mathrm{kg}$, while the lowest total concentration of $2.97 \times 10^{9} \mathrm{mg} / \mathrm{kg}$ was observed in FARO 44 . Table 3 and 4 shows average daily dose of polycyclic aromatic hydrocarbons in rice samples from different locations of Bade and Karasuwa Local Government Area. The highest average daily dose (ADD) of $5.84 \times 10^{11} \mathrm{mg} / \mathrm{kg}$ in FARO 44 was recorded at Jawa Agricultural Location, while the lowest ADD of $1.20 \times 10^{15} \mathrm{mg} / \mathrm{kg}$ in FARO 52 was recorded at Rina Kunu Agricultural Location. While for Karasuwa area, the highest average daily dose (ADD) of $1.80 \times 10^{14} \mathrm{mg} / \mathrm{kg}$ and the lowest TDD of $2.34 \times 10^{15} \mathrm{mg} / \mathrm{kg}$ were recorded in FARO 52 at Wachakal and Wachakal Ngurodi Agricultural Location, respectively.

Table 1. Concentrations of polycyclic aromatic hydrocarbon (PAHs) in rice samples from different agricultural locations, Bade Local Government area, Yobe State, Nigeria

\begin{tabular}{|c|c|c|c|c|c|c|c|c|c|c|}
\hline \multicolumn{11}{|c|}{ Concentrations (mg/kg) } \\
\hline \multirow[b]{2}{*}{ PAHS } & \multirow[b]{2}{*}{ No of ring } & \multirow[b]{2}{*}{ MACs } & \multicolumn{2}{|c|}{ Mashayan Bululu } & \multicolumn{3}{|c|}{ Jawa } & \multicolumn{3}{|c|}{ Rina Kunu } \\
\hline & & & FARO44 & FARO 52 & FARO 42 & FARO 44 & FARO 52 & FARO 45 & FARO 44 & FARO 52 \\
\hline Nap & 2 & 1 & $1.00 \times 10^{11}$ & $2.12 \times 10^{9}$ & $2.00 \times 10^{5}$ & $2.44 \times 10^{6}$ & $8.30 \times 10^{9}$ & $4.33 \times 10^{10}$ & $1.00 \times 10^{10}$ & $4.04 \times 10^{9}$ \\
\hline 2-Nap & 2 & 2 & $1.03 \times 10^{10}$ & $2.43 \times 10^{10}$ & $3.01 \times 10^{8}$ & $2.44 \times 10^{6}$ & $3.30 \times 10^{8}$ & $1.03 \times 10^{9}$ & $2.09 \times 10^{9}$ & $2.22 \times 10^{10}$ \\
\hline Acy & 3 & 3 & $2.22 \times 10^{10}$ & $1.00 \times 10^{10}$ & $3.22 \times 10^{7}$ & $3.22 \times 10^{6}$ & $2.11 \times 10^{10}$ & $3.22 \times 10^{10}$ & $4.04 \times 10^{8}$ & $1.09 \times 10^{11}$ \\
\hline Ace & 3 & 3 & $1.03 \times 10^{11}$ & $2.22 \times 10^{11}$ & $2.11 \times 10^{8}$ & $1.00 \times 10^{6}$ & $1.23 \times 10^{10}$ & $9.33 \times 10^{10}$ & $6.77 \times 10^{10}$ & $1.00 \times 10^{10}$ \\
\hline Fluo & 3 & 3 & $2.22 \times 10^{12}$ & $4.20 \times 10^{10}$ & $4.23 \times 10^{5}$ & $3.22 \times 10^{7}$ & $3.33 \times 10^{10}$ & $8.34 \times 10^{10}$ & $5.00 \times 10^{10}$ & $3.00 \times 10^{10}$ \\
\hline Phen & 3 & 3 & $1.00 \times 10^{11}$ & $5.22 \times 10^{10}$ & $3.12 \times 10^{8}$ & $3.21 \times 10^{9}$ & $3.22 \times 10^{11}$ & $3.82 \times 10^{11}$ & $4.90 \times 10^{11}$ & $1.02 \times 10^{11}$ \\
\hline Anth & 3 & 3 & $2.22 \times 10^{10}$ & $2.83 \times 10^{11}$ & $3.11 \times 10^{7}$ & $5.13 \times 10^{6}$ & $3.85 \times 10^{9}$ & $5.98 \times 10^{10}$ & $1.03 \times 10^{10}$ & $1.03 \times 10^{12}$ \\
\hline Fluo & 4 & 3 & $2.22 \times 10^{11}$ & $5.33 \times 10^{10}$ & $5.33 \times 10^{9}$ & $3.22 \times 10^{6}$ & $1.00 \times 10^{10}$ & $6.88 \times 10^{10}$ & $1.00 \times 10^{11}$ & $1.00 \times 10^{11}$ \\
\hline Py & 4 & 3 & $1.00 \times 10^{10}$ & $3.44 \times 10^{11}$ & $6.33 \times 10^{9}$ & $3.23 \times 10^{5}$ & $1.00 \times 10^{10}$ & $4.44 \times 10^{11}$ & $3.00 \times 10^{10}$ & $1.00 \times 10^{10}$ \\
\hline $\mathrm{BaA}$ & 4 & 0.15 & $2.00 \times 10^{11}$ & $9.32 \times 10^{9}$ & $1.20 \times 10^{8}$ & $6.33 \times 10^{8}$ & $1.00 \times 10^{11}$ & $5.00 \times 10^{11}$ & $1.00 \times 10^{10}$ & $1.00 \times 10^{12}$ \\
\hline Chry & 4 & - & $1.00 \times 10^{11}$ & $5.11 \times 10^{10}$ & $4.11 \times 10^{7}$ & $4.32 \times 10^{9}$ & $1.00 \times 10^{9}$ & $4.90 \times 10^{9}$ & $3.22 \times 10^{11}$ & $1.00 \times 10^{11}$ \\
\hline $\mathrm{BbF}$ & 5 & 0.3 & $2.03 \times 10^{13}$ & $2.34 \times 10^{11}$ & $4.88 \times 10^{8}$ & $4.44 \times 10^{9}$ & $4.23 \times 10^{10}$ & $3.00 \times 10^{10}$ & $4.32 \times 10^{10}$ & $1.34 \times 10^{12}$ \\
\hline $\mathrm{BkF}$ & 5 & - & $1.00 \times 10^{11}$ & $4.22 \times 10^{10}$ & $3.22 \times 10^{6}$ & $1.00 \times 10^{8}$ & $5.34 \times 10^{11}$ & $8.23 \times 10^{11}$ & $5.33 \times 10^{11}$ & $1.00 \times 10^{10}$ \\
\hline $\mathrm{BaP}$ & 5 & 0.3 & $2.04 \times 10^{10}$ & $5.11 \times 10^{10}$ & $4.00 \times 10^{8}$ & $5.55 \times 10^{6}$ & $5.34 \times 10^{10}$ & $9.99 \times 10^{10}$ & $1.04 \times 10^{11}$ & $1.05 \times 10^{10}$ \\
\hline DahA & 6 & 0.3 & $1.23 \times 10^{10}$ & $3.22 \times 10^{10}$ & $3.23 \times 10^{10}$ & $3.22 \times 10^{7}$ & $5.12 \times 10^{11}$ & $3.22 \times 10^{9}$ & $1.00 \times 10^{10}$ & $1.00 \times 10^{12}$ \\
\hline IP & 6 & - & $2.11 \times 10^{10}$ & $2.11 \times 10^{9}$ & $3.22 \times 10^{8}$ & $1.00 \times 10^{9}$ & $4.95 \times 10^{9}$ & $2.34 \times 10^{10}$ & $3.12 \times 10^{09}$ & $1.00 \times 10^{10}$ \\
\hline & Total & & $2.20 \times 10^{9}$ & $1.72 \times 10^{8}$ & $6.68 \times 10^{5}$ & $5.60 \times 10^{5}$ & $5.25 \times 10^{8}$ & $1.44 \times 10^{8}$ & $4.50 \times 10^{8}$ & $5.11 \times 10^{9}$ \\
\hline
\end{tabular}

MACs = Maximum Allowable Concentration (ATSDR, 2006) 
Table 2. Concentrations of polycyclic aromatic hydrocarbon (PAHs) in rice samples from different agricultural locations, Karasuwa Local Government area, Yobe State, Nigeria

\begin{tabular}{|c|c|c|c|c|c|c|c|c|}
\hline \multirow[b]{3}{*}{ PAHS } & \multirow[b]{3}{*}{ No of Rings } & \multirow[b]{3}{*}{ MACs } & \multicolumn{4}{|c|}{$(\mathrm{mg} / \mathrm{kg})$} & & \\
\hline & & & \multicolumn{2}{|c|}{ Wachakal } & \multicolumn{2}{|c|}{ Jaji Maji } & \multicolumn{2}{|c|}{ Wachakal Ngurodi } \\
\hline & & & FARO 44 & FARO 52 & FARO 42 & FARO 44 & FARO 44 & FARO 52 \\
\hline Nap & 2 & 1 & $1.23 \times 10^{10}$ & $4.09 \times 10^{10}$ & $2.11 \times 10^{10}$ & $4.32 \times 10^{10}$ & $4.02 \times 10^{10}$ & $1.43 \times 10^{1 \mathrm{C}}$ \\
\hline 2-Nap & 2 & 2 & $1.03 \times 10^{12}$ & $5.33 \times 10^{9}$ & $2.44 \times 10^{11}$ & $2.13 \times 10^{11}$ & $1.34 \times 10^{11}$ & $2.82 \times 10^{10}$ \\
\hline Acy & 3 & 3 & $1.00 \times 10^{10}$ & $5.43 \times 10^{10}$ & $3.01 \times 10^{10}$ & $2.02 \times 10^{10}$ & $1.02 \times 10^{10}$ & $5.34 \times 10^{11}$ \\
\hline Ace & 3 & 3 & $1.43 \times 10^{10}$ & $3.45 \times 10^{11}$ & $1.03 \times 10^{9}$ & $1.87 \times 10^{10}$ & $3.34 \times 10^{10}$ & $4.34 \times 10^{16}$ \\
\hline Fluo & 3 & 3 & $2.33 \times 10^{11}$ & $5.33 \times 10^{10}$ & $3.21 \times 10^{10}$ & $3.22 \times 10^{10}$ & $4.34 \times 10^{10}$ & $2.34 \times 10^{1 \mathrm{C}}$ \\
\hline Phen & 3 & 3 & $3.21 \times 10^{10}$ & $4.33 \times 10^{9}$ & $3.11 \times 10^{10}$ & $3.10 \times 10^{10}$ & $2.34 \times 10^{11}$ & $1.23 \times 10^{11}$ \\
\hline Anth & 3 & 3 & $3.22 \times 10^{10}$ & $6.04 \times 10^{11}$ & $5.08 \times 10^{11}$ & $3.02 \times 10^{10}$ & $2.09 \times 10^{10}$ & $1.98 \times 10^{10}$ \\
\hline Fluo & 4 & 3 & $4.22 \times 10^{10}$ & $4.34 \times 10^{10}$ & $3.11 \times 10^{10}$ & $1.22 \times 10^{10}$ & $3.34 \times 10^{10}$ & $1.98 \times 10^{11}$ \\
\hline Py & 4 & 3 & $4.44 \times 10^{10}$ & $3.23 \times 10^{9}$ & $3.11 \times 10^{10}$ & $1.87 \times 10^{10}$ & $1.09 \times 10^{11}$ & $3.34 \times 10^{10}$ \\
\hline $\mathrm{BaA}$ & 4 & 0.15 & $4.22 \times 10^{11}$ & $3.23 \times 10^{11}$ & $6.11 \times 10^{11}$ & $1.12 \times 10^{11}$ & $4.32 \times 10^{11}$ & $7.00 \times 10^{10}$ \\
\hline Chry & 4 & - & $3.23 \times 10^{10}$ & $3.34 \times 10^{10}$ & $4.01 \times 10^{10}$ & $1.98 \times 10^{10}$ & $2.23 \times 10^{10}$ & $6.34 \times 10^{11}$ \\
\hline $\mathrm{BbF}$ & 5 & 0.3 & $5.32 \times 10^{11}$ & $4.54 \times 10^{10}$ & $4.22 \times 10^{10}$ & $3.23 \times 10^{10}$ & $3.87 \times 10^{10}$ & $3.11 \times 10^{10}$ \\
\hline $\mathrm{BkF}$ & 5 & - & $1.00 \times 10^{9}$ & $9.32 \times 10^{9}$ & $1.00 \times 10^{11}$ & $2.32 \times 10^{11}$ & $1.02 \times 10^{11}$ & $8.11 \times 10^{11}$ \\
\hline $\mathrm{BaP}$ & 5 & 0.3 & $1.00 \times 10^{10}$ & $4.98 \times 10^{10}$ & $5.01 \times 10^{10}$ & $4.34 \times 10^{10}$ & $2.23 \times 10^{10}$ & $1.88 \times 10^{11}$ \\
\hline DahA & 6 & 0.3 & $2.33 \times 10^{11}$ & $3.45 \times 10^{10}$ & $2.21 \times 10^{10}$ & $2.34 \times 10^{11}$ & $1.11 \times 10^{10}$ & $1.07 \times 10^{16}$ \\
\hline IP & 6 & - & $2.22 \times 10^{10}$ & $8.98 \times 10^{11}$ & $1.19 \times 10^{11}$ & $9.23 \times 10^{10}$ & $3.23 \times 10^{10}$ & $1.76 \times 10^{15}$ \\
\hline \multicolumn{3}{|c|}{ Total } & $2.97 \times 10^{9}$ & $2.50 \times 10^{8}$ & $4.18 \times 10^{9}$ & $4.00 \times 10^{9}$ & $3.18 \times 10^{9}$ & $2.99 \times 10^{9}$ \\
\hline
\end{tabular}

MACs = Maximum Allowable Concentration (ATSDR, 2006) 
Table 3. Average daily dose $\left(\mathrm{mg} / \mathrm{kg} \mathrm{day}^{-1}\right)$ of some polycyclic aromatic hydrocarbon (PAHs) in rice samples from different agricultural locations, Bade Local Government area, Yobe State, Nigeria

\begin{tabular}{|c|c|c|c|c|c|c|c|c|}
\hline \multicolumn{9}{|c|}{$\left(\mathrm{mg} / \mathrm{kg} \mathrm{day}^{-1}\right)$} \\
\hline \multirow[b]{2}{*}{ PAHS } & \multicolumn{2}{|c|}{ Mashayan Bululu } & \multicolumn{3}{|c|}{ Jawa } & \multicolumn{3}{|c|}{ Rina Kunu } \\
\hline & FARO 44 & FARO 52 & FARO 42 & FARO 44 & FARO 52 & FARO 45 & FARO 44 & FARO 52 \\
\hline Naphthalene & $9.79 \times 10^{20}$ & $2.07 \times 10^{17}$ & $1.96 \times 10^{13}$ & $1.96 \times 10^{14}$ & $8.12 \times 10^{17}$ & $4.24 \times 10^{18}$ & $9.79 \times 10^{19}$ & $3.95 \times 10^{17}$ \\
\hline 2-Methyl Naphthalene & $1.01 \times 10^{17}$ & $2.38 \times 10^{18}$ & $2.95 \times 10^{16}$ & $1.96 \times 10^{14}$ & $3.23 \times 10^{16}$ & $1.01 \times 10^{17}$ & $2.05 \times 10^{17}$ & $2.17 \times 10^{18}$ \\
\hline Acenapthylene & $2.17 \times 10^{18}$ & $9.79 \times 10^{19}$ & $3.15 \times 10^{15}$ & $2.94 \times 10^{14}$ & $2.06 \times 10^{18}$ & $3.15 \times 10^{18}$ & $3.95 \times 10^{16}$ & $1.07 \times 10^{19}$ \\
\hline Acenaphthene & $1.01 \times 10^{19}$ & $2.17 \times 10^{19}$ & $2.06 \times 10^{16}$ & $9.79 \times 10^{15}$ & $1.20 \times 10^{18}$ & $9.13 \times 10^{18}$ & $6.62 \times 10^{18}$ & $9.79 \times 10^{19}$ \\
\hline Fluorene & $2.17 \times 10^{20}$ & $4.11 \times 10^{18}$ & $4.11 \times 10^{13}$ & $3.15 \times 10^{15}$ & $3.26 \times 10^{18}$ & $8.16 \times 10^{18}$ & $4.89 \times 10^{18}$ & $2.94 \times 10^{18}$ \\
\hline Phenanthrene & $9.79 \times 10^{20}$ & $5.11 \times 10^{18}$ & $3.05 \times 10^{16}$ & $3.14 \times 10^{17}$ & $3.15 \times 10^{19}$ & $3.74 \times 10^{19}$ & $4.80 \times 10^{19}$ & $9.98 \times 10^{20}$ \\
\hline Anthracene & $2.17 \times 10^{17}$ & $2.77 \times 10^{18}$ & $2.94 \times 10^{14}$ & $4.99 \times 10^{13}$ & $3.77 \times 10^{16}$ & $5.85 \times 10^{17}$ & $1.01 \times 10^{17}$ & $1.01 \times 10^{19}$ \\
\hline Fluoranthene & $2.17 \times 10^{19}$ & $5.22 \times 10^{18}$ & $5.22 \times 10^{17}$ & $2.94 \times 10^{14}$ & $9.79 \times 10^{19}$ & $6.73 \times 10^{18}$ & $9.79 \times 10^{20}$ & $9.79 \times 10^{20}$ \\
\hline Pyrene & $9.79 \times 10^{19}$ & $3.37 \times 10^{19}$ & $6.19 \times 10^{17}$ & $3.13 \times 10^{13}$ & $9.79 \times 10^{19}$ & $4.34 \times 10^{19}$ & $2.94 \times 10^{18}$ & $9.79 \times 10^{19}$ \\
\hline Benz(a)Anthracene & $1.96 \times 10^{17}$ & $9.12 \times 10^{15}$ & $9.79 \times 10^{15}$ & $5.87 \times 10^{14}$ & $9.79 \times 10^{18}$ & $4.89 \times 10^{17}$ & $9.79 \times 10^{17}$ & $9.79 \times 10^{19}$ \\
\hline Chrysene & $9.79 \times 10^{20}$ & $5.00 \times 10^{18}$ & $4.02 \times 10^{15}$ & $4.23 \times 10^{17}$ & $9.79 \times 10^{18}$ & $4.80 \times 10^{17}$ & $3.15 \times 10^{19}$ & $9.79 \times 10^{20}$ \\
\hline Benz(b)Fluoranthene & $1.99 \times 10^{19}$ & $2.29 \times 10^{17}$ & $3.91 \times 10^{14}$ & $4.34 \times 10^{15}$ & $4.14 \times 10^{16}$ & $2.94 \times 10^{16}$ & $4.23 \times 10^{16}$ & $1.31 \times 10^{18}$ \\
\hline Benz(k)Fluoranthene & $9.79 \times 10^{18}$ & $4.13 \times 10^{16}$ & $3.15 \times 10^{12}$ & $9.79 \times 10^{15}$ & $5.23 \times 10^{17}$ & $8.05 \times 10^{17}$ & $5.22 \times 10^{17}$ & $9.79 \times 10^{17}$ \\
\hline Benz(a)Pyrene & $1.99 \times 10^{15}$ & $5.00 \times 10^{15}$ & $3.91 \times 10^{13}$ & $5.43 \times 10^{11}$ & $5.23 \times 10^{15}$ & $9.78 \times 10^{15}$ & $1.02 \times 10^{16}$ & $1.03 \times 10^{15}$ \\
\hline Dibenz(a,h)Anthracene & $1.20 \times 10^{15}$ & $3.15 \times 10^{15}$ & $3.16 \times 10^{15}$ & $3.15 \times 10^{12}$ & $5.01 \times 10^{16}$ & $3.15 \times 10^{14}$ & $9.79 \times 10^{16}$ & $9.79 \times 10^{18}$ \\
\hline Indinol(1,2,3-cd)Pyrene & $2.06 \times 10^{17}$ & $2.06 \times 10^{16}$ & $3.15 \times 10^{15}$ & $9.79 \times 10^{17}$ & $4.84 \times 10^{16}$ & $2.29 \times 10^{17}$ & $3.05 \times 10^{28}$ & $9.79 \times 10^{18}$ \\
\hline TDD & $3.28 \times 10^{15}$ & $1.80 \times 10^{15}$ & $4.24 \times 10^{15}$ & $5.84 \times 10^{15}$ & $7.49 \times 10^{15}$ & $4.19 \times 10^{14}$ & $2.10 \times 10^{15}$ & $1.20 \times 10^{15}$ \\
\hline
\end{tabular}


Table 4. Average daily dose $\left(\mathrm{mg} / \mathrm{kg} \mathrm{day}^{-1}\right)$ of some polycyclic aromatic hydrocarbon (PAHs) in rice samples from different agricultural locations, Karasuwa Local Government area, Yobe State, Nigeria

\begin{tabular}{|c|c|c|c|c|c|c|}
\hline \multicolumn{7}{|c|}{$\left(\mathrm{mg} / \mathrm{kg} \mathrm{day}^{-1}\right)$} \\
\hline \multirow[b]{2}{*}{ PAHS } & \multicolumn{2}{|c|}{ Wachakal } & \multicolumn{2}{|c|}{ Jaji Maji } & \multicolumn{2}{|c|}{ Wachakal Ngurodi } \\
\hline & FARO 44 & FARO 52 & FARO 44 & FARO 52 & FARO 44 & FARO 52 \\
\hline Naphthalene & $1.20 \times 10^{18}$ & $4.00 \times 10^{18}$ & $2.06 \times 10^{18}$ & $4.23 \times 10^{18}$ & $3.93 \times 10^{18}$ & $1.40 \times 10^{18}$ \\
\hline 2-Methyl Naphthalene & $1.01 \times 10^{20}$ & $5.22 \times 10^{17}$ & $2.39 \times 10^{19}$ & $2.08 \times 10^{19}$ & $1.31 \times 10^{19}$ & $2.76 \times 10^{18}$ \\
\hline Acenapthylene & $9.79 \times 10^{19}$ & $5.31 \times 10^{18}$ & $2.95 \times 10^{19}$ & $1.98 \times 10^{18}$ & $9.98 \times 10^{19}$ & $5.23 \times 10^{19}$ \\
\hline Acenaphthene & $1.40 \times 10^{18}$ & $3.38 \times 10^{19}$ & $1.01 \times 10^{17}$ & $1.83 \times 10^{18}$ & $3.27 \times 10^{18}$ & $4.25 \times 10^{18}$ \\
\hline Fluorene & $2.28 \times 10^{19}$ & $5.22 \times 10^{18}$ & $3.14 \times 10^{18}$ & $3.15 \times 10^{18}$ & $4.25 \times 10^{18}$ & $2.29 \times 10^{18}$ \\
\hline Phenanthrene & $3.14 \times 10^{18}$ & $4.24 \times 10^{17}$ & $3.04 \times 10^{18}$ & $3.03 \times 10^{18}$ & $2.29 \times 10^{19}$ & $1.20 \times 10^{19}$ \\
\hline Anthracene & $3.15 \times 10^{17}$ & $5.90 \times 10^{18}$ & $4.97 \times 10^{18}$ & $2.96 \times 10^{17}$ & $2.05 \times 10^{17}$ & $1.94 \times 10^{17}$ \\
\hline Fluoranthene & $4.13 \times 10^{18}$ & $4.25 \times 10^{18}$ & $3.04 \times 10^{18}$ & $1.19 \times 10^{18}$ & $3.27 \times 10^{18}$ & $1.94 \times 10^{19}$ \\
\hline Pyrene & $4.34 \times 10^{18}$ & $3.16 \times 10^{17}$ & $3.04 \times 10^{18}$ & $1.83 \times 10^{18}$ & $1.07 \times 10^{19}$ & $3.27 \times 10^{18}$ \\
\hline Benz(a)Anthracene & $4.13 \times 10^{17}$ & $3.16 \times 10^{17}$ & $5.98 \times 10^{17}$ & $1.10 \times 10^{18}$ & $4.23 \times 10^{17}$ & $6.85 \times 10^{16}$ \\
\hline Chrysene & $3.16 \times 10^{18}$ & $3.27 \times 10^{19}$ & $3.92 \times 10^{18}$ & $1.94 \times 10^{18}$ & $2.18 \times 10^{18}$ & $6.20 \times 10^{19}$ \\
\hline Benz(b)Fluoranthene & $5.21 \times 10^{17}$ & $4.44 \times 10^{16}$ & $4.22 \times 10^{16}$ & $3.16 \times 10^{16}$ & $3.79 \times 10^{16}$ & $3.04 \times 10^{16}$ \\
\hline Benz(k)Fluoranthene & $9.79 \times 10^{16}$ & $9.12 \times 10^{15}$ & $9.79 \times 10^{18}$ & $2.27 \times 10^{17}$ & $9.98 \times 10^{18}$ & $7.94 \times 10^{17}$ \\
\hline Benz(a)Pyrene & $9.79 \times 10^{16}$ & $4.87 \times 10^{15}$ & $4.90 \times 10^{15}$ & $4.28 \times 10^{15}$ & $2.18 \times 10^{15}$ & $1.84 \times 10^{16}$ \\
\hline Dibenz(a,h)Anthracene & $2.28 \times 10^{16}$ & $3.38 \times 10^{15}$ & $2.16 \times 10^{15}$ & $2.29 \times 10^{16}$ & $1.09 \times 10^{15}$ & $1.05 \times 10^{15}$ \\
\hline Indinol(1,2,3-cd)Pyrene & $2.17 \times 10^{17}$ & $8.79 \times 10^{18}$ & $1.16 \times 10^{18}$ & $9.03 \times 10^{17}$ & $3.16 \times 10^{17}$ & $1.72 \times 10^{26}$ \\
\hline TDD & $2.35 \times 10^{15}$ & $1.80 \times 10^{14}$ & $7.59 \times 10^{15}$ & $4.99 \times 10^{15}$ & $3.77 \times 10^{15}$ & $2.34 \times 10^{15}$ \\
\hline
\end{tabular}

Table 5 and 6 present the hazard quotient and hazard index of non-carcinogenic polycyclic aromatic hydrocarbons via consumption of rice from different locations of Bade and Karasuwa Local Government Area. The highest hazard index (HI) of $2.04 \times 10^{11} \mathrm{mg} / \mathrm{kg}$ was recorded in FARO 42 at Jawa Location, while the lowest hazard index of $7.34 \times 10^{16} \mathrm{mg} / \mathrm{kg}$ was recorded in FARO 44 at Mashayan Bululu Location. For Karasuwa area, the highest hazard index (HI) of $5.45 \times 10^{15} \mathrm{mg} / \mathrm{kg}$ was recorded in FARO 52 at Wachakal Location, while the lowest hazard index of $4.80 \times 10^{16} \mathrm{mg} / \mathrm{kg}$ was recorded in FARO 52 at Wachakal Ngurodi Location. 
Table 5. Hazard quotient and hazard index $\left(\mathrm{mg} / \mathrm{kg}\right.$ day $\left.^{-1}\right)$ of non-carcinogenic polycyclic aromatic hydrocarbon (PAHs) via consumption of rice from different agricultural locations, Bade Local Government area, Yobe State, Nigeria

\begin{tabular}{|c|c|c|c|c|c|c|c|c|}
\hline \multirow[b]{3}{*}{ PAHS } & \multicolumn{8}{|c|}{$\left(\mathrm{mg} / \mathrm{kg} \mathrm{day}^{-1}\right)$} \\
\hline & \multicolumn{2}{|c|}{ Mashayan Bululu } & \multicolumn{3}{|c|}{ Jawa } & \multicolumn{3}{|c|}{ Rina Kunu } \\
\hline & FARO 44 & FARO 52 & FARO 42 & FARO 44 & FARO 52 & FARO 45 & FARO 44 & FARO 52 \\
\hline Naphthalene & $4.90 \times 10^{18}$ & $1.04 \times 10^{15}$ & $9.80 \times 10^{12}$ & $9.80 \times 10^{13}$ & $4.06 \times 10^{15}$ & $2.12 \times 10^{16}$ & $4.90 \times 10^{17}$ & $1.98 \times 10^{15}$ \\
\hline 2-Methyl Naphthalene & $5.05 \times 10^{16}$ & $1.19 \times 10^{16}$ & $1.48 \times 10^{14}$ & $9.80 \times 10^{13}$ & $1.62 \times 10^{14}$ & $5.05 \times 10^{16}$ & $1.03 \times 10^{15}$ & $1.09 \times 10^{16}$ \\
\hline Acenapthylene & $1.09 \times 10^{16}$ & $4.90 \times 10^{17}$ & $1.58 \times 10^{13}$ & $1.47 \times 10^{12}$ & $1.03 \times 10^{16}$ & $1.58 \times 10^{16}$ & $1.98 \times 10^{14}$ & $5.35 \times 10^{18}$ \\
\hline Acenaphthene & $1.68 \times 10^{18}$ & $3.62 \times 10^{18}$ & $3.43 \times 10^{15}$ & $1.63 \times 10^{13}$ & $2.00 \times 10^{17}$ & $1.52 \times 10^{16}$ & $1.10 \times 10^{16}$ & $1.63 \times 10^{17}$ \\
\hline Fluorene & $5.43 \times 10^{19}$ & $1.03 \times 10^{16}$ & $1.03 \times 10^{11}$ & $7.88 \times 10^{14}$ & $8.15 \times 10^{17}$ & $2.04 \times 10^{16}$ & $1.22 \times 10^{16}$ & $7.35 \times 10^{17}$ \\
\hline Phenanthrene & $2.45 \times 10^{18}$ & $1.28 \times 10^{16}$ & $7.63 \times 10^{15}$ & $7.85 \times 10^{16}$ & $7.88 \times 10^{18}$ & $9.35 \times 10^{18}$ & $1.20 \times 10^{17}$ & $2.50 \times 10^{18}$ \\
\hline Anthracene & $7.23 \times 10^{17}$ & $9.23 \times 10^{18}$ & $9.80 \times 10^{14}$ & $1.66 \times 10^{12}$ & $1.26 \times 10^{15}$ & $1.95 \times 10^{16}$ & $3.37 \times 10^{17}$ & $3.37 \times 10^{19}$ \\
\hline Fluoranthene & $5.43 \times 10^{18}$ & $1.31 \times 10^{16}$ & $1.31 \times 10^{15}$ & $7.35 \times 10^{13}$ & $2.45 \times 10^{17}$ & $1.68 \times 10^{16}$ & $2.45 \times 10^{18}$ & $2.45 \times 10^{18}$ \\
\hline Pyrene & $3.26 \times 10^{17}$ & $1.12 \times 10^{17}$ & $2.06 \times 10^{15}$ & $1.04 \times 10^{11}$ & $3.26 \times 10^{17}$ & $1.45 \times 10^{17}$ & $9.80 \times 10^{17}$ & $3.26 \times 10^{17}$ \\
\hline Hazard Index (HI) & $7.34 \times 10^{16}$ & $1.59 \times 10^{15}$ & $2.04 \times 10^{11}$ & $1.65 \times 10^{11}$ & $2.18 \times 10^{14}$ & $1.62 \times 10^{15}$ & $2.12 \times 10^{14}$ & $2.22 \times 10^{15}$ \\
\hline
\end{tabular}

Table 6. Hazard quotient and hazard index $\left(\mathrm{mg} / \mathrm{kg}\right.$ day $\left.{ }^{-1}\right)$ of non-carcinogenic polycyclic aromatic hydrocarbon (PAHs) via consumption of rice from different agricultural locations, Karasuwa Local Government area, Yobe State, Nigeria

\begin{tabular}{|c|c|c|c|c|c|c|}
\hline \multicolumn{7}{|c|}{$\left(\mathrm{mg} / \mathrm{kg} \mathrm{day}^{-1}\right)$} \\
\hline \multirow[b]{2}{*}{ PAHS } & \multicolumn{2}{|c|}{ Wachakal } & \multicolumn{2}{|c|}{ Jaji Maji } & \multicolumn{2}{|c|}{ Wachakal Ngurodi } \\
\hline & FARO 44 & FARO 52 & FARO 44 & FARO 52 & FARO 44 & FARO 52 \\
\hline Naphthalene & $6.00 \times 10^{17}$ & $2.00 \times 10^{16}$ & $1.03 \times 10^{16}$ & $2.12 \times 10^{16}$ & $1.97 \times 10^{16}$ & $7.00 \times 10^{17}$ \\
\hline 2-Methyl Naphthalene & $5.05 \times 10^{16}$ & $2.61 \times 10^{15}$ & $1.20 \times 10^{17}$ & $1.04 \times 10^{17}$ & $6.55 \times 10^{18}$ & $1.38 \times 10^{16}$ \\
\hline Acenapthylene & $4.90 \times 10^{17}$ & $2.66 \times 10^{16}$ & $1.48 \times 10^{16}$ & $9.90 \times 10^{17}$ & $4.90 \times 10^{17}$ & $2.62 \times 10^{17}$ \\
\hline Acenaphthene & $2.33 \times 10^{17}$ & $5.63 \times 10^{18}$ & $1.68 \times 10^{16}$ & $3.05 \times 10^{17}$ & $5.45 \times 10^{17}$ & $7.08 \times 10^{17}$ \\
\hline Fluorene & $5.70 \times 10^{18}$ & $1.31 \times 10^{16}$ & $7.85 \times 10^{17}$ & $7.90 \times 10^{17}$ & $1.06 \times 10^{16}$ & $5.73 \times 10^{17}$ \\
\hline Phenanthrene & $7.85 \times 10^{17}$ & $1.06 \times 10^{15}$ & $7.60 \times 10^{17}$ & $7.60 \times 10^{17}$ & $5.73 \times 10^{18}$ & $3.00 \times 10^{18}$ \\
\hline Anthracene & $1.05 \times 10^{16}$ & $1.97 \times 10^{17}$ & $1.66 \times 10^{17}$ & $9.87 \times 10^{17}$ & $6.83 \times 10^{17}$ & $6.47 \times 10^{19}$ \\
\hline Fluoranthene & $1.03 \times 10^{16}$ & $1.06 \times 10^{16}$ & $7.60 \times 10^{17}$ & $2.98 \times 10^{17}$ & $8.18 \times 10^{17}$ & $4.85 \times 10^{18}$ \\
\hline Pyrene & $1.45 \times 10^{16}$ & $1.05 \times 10^{15}$ & $1.01 \times 10^{16}$ & $6.10 \times 10^{17}$ & $3.57 \times 10^{18}$ & $1.09 \times 10^{16}$ \\
\hline Hazard Index (HI) & $5.70 \times 10^{16}$ & $5.45 \times 10^{15}$ & $7.79 \times 10^{16}$ & $6.96 \times 10^{16}$ & $5.72 \times 10^{16}$ & $4.80 \times 10^{16}$ \\
\hline
\end{tabular}

Table 7 and 8 shows carcinogenic risk assessment of polycyclic aromatic hydrocarbons in rice samples from Bade and Karasuwa Local Government Area. The highest total incremental lifetime expectancy cancer risk (ILECR) of 4.19E-10 mg/ was recorded $\mathrm{kg}$ in FARO 44 at Jawa location, while the lowest total ILECR of $7.61 \mathrm{E}-15 \mathrm{mg} / \mathrm{kg}$ was recorded in FARO 52 at Rina Kunu location, while for Karasuwa area, the highest total incremental lifetime expectancy cancer risk (ILECR) of $6.13 \times 10^{14} \mathrm{mg} / \mathrm{kg}$ was observed in FARO 52, while the lowest total ILECR of $8.97 \times 10^{15} \mathrm{mg} / \mathrm{kg}$ was recorded in FARO 44 for Wachakal location. 
Table 7. Carcinogenic risk assessment $\left(\mathrm{mg} / \mathrm{kg} \mathrm{day}^{-1}\right)$ of some polycyclic aromatic hydrocarbon (PAHs) in rice samples from different agricultural locations, Bade Local Government area, Yobe State, Nigeria

\begin{tabular}{|c|c|c|c|c|c|c|c|c|}
\hline \multirow[b]{3}{*}{ PAHS } & \multicolumn{8}{|c|}{$\left(\mathrm{mg} / \mathrm{kg} \mathrm{day}^{-1}\right)$} \\
\hline & \multicolumn{2}{|c|}{ Mashayan Bululu } & \multicolumn{3}{|c|}{ Jawa } & \multicolumn{3}{|c|}{ Rina Kunu } \\
\hline & FARO 44 & FARO 52 & FARO 42 & FARO 44 & FARO 52 & FARO 45 & FARO 44 & FARO 52 \\
\hline Benz(a)Anthracene & $1.43 \times 10^{17}$ & $6.66 \times 10^{15}$ & $7.15 \times 10^{15}$ & $4.29 \times 10^{14}$ & $7.15 \times 10^{18}$ & $3.57 \times 10^{17}$ & $7.15 \times 10^{17}$ & $7.15 \times 10^{19}$ \\
\hline Chrysene & $7.15 \times 10^{22}$ & $3.65 \times 10^{15}$ & $2.93 \times 10^{17}$ & $3.09 \times 10^{19}$ & $7.15 \times 10^{20}$ & $3.50 \times 10^{19}$ & $2.30 \times 10^{21}$ & $7.15 \times 10^{22}$ \\
\hline Benz(b)Fluoranthene & $1.45 \times 10^{19}$ & $1.67 \times 10^{17}$ & $2.85 \times 10^{14}$ & $3.17 \times 10^{15}$ & $3.02 \times 10^{20}$ & $2.15 \times 10^{16}$ & $3.09 \times 10^{16}$ & $9.56 \times 10^{19}$ \\
\hline Benz(k)Fluoranthene & $7.15 \times 10^{19}$ & $3.01 \times 10^{17}$ & $2.30 \times 10^{13}$ & $7.15 \times 10^{16}$ & $3.82 \times 10^{18}$ & $5.88 \times 10^{18}$ & $3.81 \times 10^{18}$ & $7.15 \times 10^{18}$ \\
\hline Benz(a)Pyrene & $1.45 \times 10^{14}$ & $3.65 \times 10^{14}$ & $2.85 \times 10^{12}$ & $3.96 \times 10^{10}$ & $3.82 \times 10^{14}$ & $7.14 \times 10^{14}$ & $7.45 \times 10^{16}$ & $7.52 \times 10^{15}$ \\
\hline Dibenz(a,h)Anthracene & $8.76 \times 10^{15}$ & $2.30 \times 10^{14}$ & $2.31 \times 10^{14}$ & $2.30 \times 10^{11}$ & $3.66 \times 10^{15}$ & $2.30 \times 10^{13}$ & $7.15 \times 10^{15}$ & $7.15 \times 10^{17}$ \\
\hline Indinol (1,2,3-cd)Pyrene & $1.50 \times 10^{17}$ & $1.50 \times 10^{16}$ & $2.30 \times 10^{15}$ & $7.15 \times 10^{17}$ & $3.53 \times 10^{16}$ & $1.67 \times 10^{17}$ & $2.23 \times 10^{26}$ & $7.15 \times 10^{18}$ \\
\hline इILECR & $2.33 \times 10^{14}$ & $6.99 \times 10^{14}$ & $3.14 \times 10^{12}$ & $4.19 \times 10^{10}$ & $4.22 \times 10^{14}$ & $3.02 \times 10^{13}$ & $8.28 \times 10^{15}$ & $7.61 \times 10^{15}$ \\
\hline
\end{tabular}

Table 8. Carcinogenic risk assessment $\left(\mathrm{mg} / \mathrm{kg} \mathrm{day}^{-1}\right)$ of some polycyclic aromatic hydrocarbon (PAHs) in rice samples from different agricultural locations, Karasuwa Local Government area, Yobe State, Nigeria

\begin{tabular}{|c|c|c|c|c|c|c|}
\hline \multicolumn{7}{|c|}{$\left(\mathrm{mg} / \mathrm{kg}\right.$ day $\left.^{-1}\right)$} \\
\hline \multirow[b]{2}{*}{ PAHS } & \multicolumn{2}{|c|}{ Wachakal } & \multicolumn{2}{|c|}{ Jaji Maji } & \multicolumn{2}{|c|}{ Wachakal Ngurodi } \\
\hline & FARO 44 & FARO 52 & FARO 44 & FARO 52 & FARO 44 & FARO 52 \\
\hline Benz(a)Anthracene & $3.01 \times 10^{17}$ & $2.31 \times 10^{17}$ & $4.37 \times 10^{17}$ & $8.03 \times 10^{19}$ & $3.09 \times 10^{17}$ & $5.00 \times 10^{16}$ \\
\hline Chrysene & $2.31 \times 10^{20}$ & $2.39 \times 10^{21}$ & $2.86 \times 10^{20}$ & $1.42 \times 10^{20}$ & $1.59 \times 10^{20}$ & $4.53 \times 10^{21}$ \\
\hline Benz(b)Fluoranthene & $3.80 \times 10^{17}$ & $3.24 \times 10^{16}$ & $3.08 \times 10^{16}$ & $2.31 \times 10^{16}$ & $2.77 \times 10^{16}$ & $2.22 \times 10^{16}$ \\
\hline Benz(k)Fluoranthene & $7.15 \times 10^{17}$ & $6.66 \times 10^{16}$ & $7.15 \times 10^{19}$ & $1.66 \times 10^{18}$ & $7.29 \times 10^{19}$ & $5.80 \times 10^{18}$ \\
\hline Benz(a)Pyrene & $7.15 \times 10^{15}$ & $3.56 \times 10^{14}$ & $3.58 \times 10^{14}$ & $3.12 \times 10^{14}$ & $1.59 \times 10^{14}$ & $1.34 \times 10^{15}$ \\
\hline Dibenz(a,h)Anthracene & $1.66 \times 10^{15}$ & $2.47 \times 10^{14}$ & $1.58 \times 10^{14}$ & $1.67 \times 10^{15}$ & $7.96 \times 10^{15}$ & $7.67 \times 10^{15}$ \\
\hline Indinol(1,2,3-cd)Pyrene & $1.58 \times 10^{17}$ & $6.42 \times 10^{18}$ & $8.47 \times 10^{19}$ & $6.59 \times 10^{17}$ & $2.31 \times 10^{17}$ & $1.26 \times 10^{26}$ \\
\hline इILECR & $8.97 \times 10^{15}$ & $6.13 \times 10^{14}$ & $5.20 \times 10^{14}$ & $3.32 \times 10^{14}$ & $2.42 \times 10^{14}$ & $9.74 \times 10^{15}$ \\
\hline
\end{tabular}

\section{Discussion}

Sixteen PAHs were detected in different variety of rice (FARO 44, 52, 42 and 45) samples from the six agricultural locations (Mashayan Bululu, Jawa, Rina Kunu, Wachakal, Jaji Maji and Wachakal Ngurodi). The lower molecular weight PAHs were observed to be the most predominant over the higher molecular weight PAHs in all the six above-mentioned agricultural locations, with PAHs such as naphthalene, fluorene and pyrene having the highest values of $2.25 \times 10^{5}, 4.26 \times 10^{5}$ and $3.23 \times 10^{5} \mathrm{mg} / \mathrm{kg}$, respectively. FARO 42 with a value of $6.68 \mathrm{E}-05$ $\mathrm{mg} / \mathrm{kg}$ in Jawa agricultural location has the highest PAHs concentration, whereas the lowest PAH concentration value of $2.20 \times 10^{9} \mathrm{mk} / \mathrm{kg}$ in FARO 44 was observed from Mashayan Bululu agricultural location.

Intake of polycyclic aromatic hydrocarbons has been estimated in many European countries (Scientific Committee on Food, 2002). According to several studies, the major dietary contributors are rice, cereals, oils and vegetables, although polycyclic aromatic hydrocarbons levels in rice are often low (Dennis et al., 1983; De-Vos et al., 1990; Tao et al., 2006). Dietary intake of food contaminants depends on both the nutritional habit of the examined population group and the concentration of contaminants in the food. All the computations in the current study were based on the mean concentration of PAHs obtained from analysis of different variety of rice (FARO 44, 52, 42 and 45) from Mashayan Bululu, Jawa, Rina Kunu, Wachakal, Jaji Maji and Wachakal Ngurodi agricultural locations. The daily dose values in all the variety of rice samples were observed to be lower than the exposure limit set by WHO $(0.004 \mathrm{mg} / \mathrm{kg})$ for an average of $70 \mathrm{~kg}$ body weight (WHO, 2006). The daily dose values from the present study were lower when compared to dietary intake of PAHs in Spanish population as described by Raquel et al. (2005) with value of $3.24 \times 10^{2} \mathrm{ug} / \mathrm{kg}$. 
The non-cancer hazard quotient assumes that there is a level of exposure below which it is unlikely that even sensitive populations would experience adverse health effects (USEPA, 1989). Results obtained for hazard quotient and index were far less than 1, the result of this study shows that consumption of rice from the said study locations have no considerable health risk based on non-carcinogenic risk as per the United States Environmental Protection Agency guidelines. Similar results were reported for PAHs intake by the general population in Estonia (Reinik et al., 2011). The average daily dose and the slope factor were used in the evaluation of the cancer risk assessment. Carcinogenic risks are estimated as the incremental probability of an individual developing cancer over a lifetime as a result of exposure to the potential carcinogen. The collective distribution of designated incremental lifetime expectancy cancer risk (ILECR) values for different variety of rice samples from the aforesaid agricultural locations in Bade and Karasuwa Local Government Areas has an ILECR values higher than Karasuwa Local Government Area. Based on the permissible limits or acceptable risk levels defined by US EPA, the upper and lower acceptable risk thresholds are represented by one in four thousand $(10-4 \mathrm{mg} / \mathrm{kg} /$ day $)$ and one in a million $(10-6 \mathrm{mg} / \mathrm{kg} /$ day) level over an average lifetime of 70 years respectively. The upper and lower thresholds indicate considerable and acceptable risk levels. Results from the present study shows that all the investigated rice samples were below the permissible limits for PAHs in adults. These levels would not present any considerable health risks upon the consumption the study rice.

\section{Conclusion}

The concentrations of PAHs in the study rice samples were lower than the maximum allowable concentration (MAC). Result from hazard quotient and hazard index of all the PAHs were $<1$ in all the rice collected from the different locations. Therefore, there would be no apparent health risk to the residents of this area as a result of consumption of rice. The incremental lifetime expectancy cancer risk shows that FARO 44 from Jawa and FARO 52 from Wachakal had the highest values, while the lowest values were recorded for FARO 52 from Rina Kuna and FARO 44 from Wachakal. Results from the present study shows that the rice samples were safe for human consumption and not significantly contaminated by PAHs.

\section{References}

Adebayo, R. A., \& Salawu, E. O. (2010). Response of Some Selected Rice Varieties to Infestation by a Root-Knot Nematode, Meloidogyne incognita, Chitwood. International Journal of Agricultural Research, 5, 453-459. https://doi.org/10.3923/ijar.2010.453.459

Akan, J. C., Mohammed, Z., Mohammed, A. I., \& Jafiya, L. (2017). Source Identification and Ecotoxicological Risk Assessment of Polycyclic Aromatic Hydrocarbons in Sediment from Komadugu River Basin, Yobe State, Nigeria. Canadian Journal of Pure and Applied Sciences, 11(3), 4355-4365.

ATSDR (Agency for Toxic Substance and Disease Registry). (2006). Toxicology Profile for Cyanide, Atlanta, GA. USA.

Benson, N. U., Anake, W. U., Adedapo, A. E., Fred-Ahmadu, O. H., \& Eke, K.P. (2016). Polycyclic aromatic hydrocarbons in imported Sardinops sagax: levels and health risk assessments through dietary exposure in Nigeria. J. Food Compos. Anal., 57, 109-116. https://doi.org/10.1016/j.jfca.2016.12.024

Benson, N. U., Williams, A. B., Anake, W. U., Eke, K. P., Adedapo, A. E., \& Olajire, A. A. (2016). Carcinogenicity and mutagenicity assessments of dietary exposure to PAHs in imported fish products in Nigeria. In G. Sorial, \& J. Hong (Eds.), Proceedings, $8^{\text {th }}$ International Conference on Environmental Science and Technology (Vol. 1., pp. 388). American Science Press, Houston, USA.

Dennis M. J., Massey, R. C., McWeeny, D. J., Knowles, M. E., \& Watson, D. (1983). Analysis of polycyclic aromatic hydrocarbons in the UK total diet. Food and Chemical Toxicology, 21, 569-574. https://doi.org/10.1016/0278-6915(83)90142-4

De-Vos, R. H., Dokkum, W., Schouten, A., \& Jomg-Berkhout, P. (1990). Polycyclic aromatic hydrocarbons in Dutch total diet samples. Food Chemistry and Toxicology, 28, 263-268. https://doi.org/10.1016/0278-6915(90)90038-O

Essumang, D. K. (2014). Polycyclic aromatic hydrocarbon (pah) in cooked (charred) rice: levels, risk assessment and source characterization. Journal of Basic \& Applied Sciences, 1(2), 63-83.

FAO. (Food Agriculture Organisation). (1972). The state of Food and Agriculture.

FAO. (Food and Agriculture Organization). (2008). Fisheries the state of world fisheries and aquaculture (SOFIA) part 1. World review of fisheries and aquaculture; fish consumption, pp. 58-65.

Hao, X., Li, J., \& Yao, Z. (2015). Changes in PAHs levels in edible oils during deep-frying process. Food Control, 
66, 233-240. https://doi.org/10.1016/j.foodcont.2016.02.012

Ito, S., \& Ishikawa, Y. (2004). Marketing of Value-Added Rice Products in Japan: Germinated Brown Rice and Rice Bread. Tottori University, Japan. FAO. International Rice Symposium, Rome, Italy.

Klein, S. J. (2014). Acenaphthene. In P. Wexler (Ed.), Encyclopedia of Toxicology (3rd ed., pp. 17-19). Oxford: Academic Press. https://doi.org/10.1016/B978-0-12-386454-3.00214-1

Li, J., Dong, H., Li, X., Han, B., Zhu, C., \& Zhang, D. (2015). Quantitatively assessing the health risk of exposure to PAHs from intake of smoked meats. Ecotoxicol. Environ. Saf., 124, 91-95. https://doi.org/10.1016/j.ecoenv.2015.10.007

Masih, A., \& Lal, J. K. (2014). Concentrations and carcinogenicprofiles of polycyclic aromatic hydrocarbons (PAHs) in groundwater of an urban site at a terai belt of North India. International Journal of Applied Engineering Research, 9(1), 1-7.

Nsikak, U. B., Omowunmi, H. F., Joseph, A.O. O., Winifred, U. A., Adebusayo, E. A., \& Abass A. (2018). Concentrations, sources and risk characterisation of polycyclic aromatic hydrocarbons (PAHs) in green, herbal and black tea products in Nigeria. Journal of Food Composition and Analysis, 66, 13-22. https://doi.org/10.1016/j.jfca.2017.11.003

Nwaichi, E., \& Ntorgbo, S. (2016). Assessment of PAHs levels in some fish and seafood from different coastal waters in the Niger Delta. Toxicol. Rep., 3, 167-172. https://doi.org/10.1016/j.toxrep.2016.01.005

Raquel, I., Antonio, A., Antonio, B., Paula, J., Maria, J. T., Maria, J. S., ... Carlos, A. G. (2005). Dietary intake of PAHs in a Spanish population. Journal of Food Protection, 68(10), 2190-2195. https://doi.org/10.4315/0362-028X-68.10.2190

Reinik, M., Tarje, T., Mati, R., Kadrin, J., Toomas, T., \& Alida, K. (2011). PAHs in meat products and estimated PAH intake by general population inEstomia. Food Additives and Conataminants, 24(4), 429-437. https://doi.org/10.1080/02652030601182862

SCF (Scientific Committee on Food). (2002). SCF/CS/CNTM/PAH/29 ADDI Final; Polycyclic aromatic hydrocarbons occurrence in foods, dietary exposure and health effects. Background document to the opinion of the Scientific Committee on Food on the risks to human health of polycyclic aromatic hydrocarbons in food. Brussels.

Sharma, T. (2014). In silico investigation of polycyclic aromatic hydrocarbons against bacterial 1-2 dioxygenase. Journal of Chemical and Pharmaceutical Research, 6, 873-877.

Tao, S., Jiao, X. C., Chen, S. H., Liu, W. X., Coveney, R. M., Zhu, L. Z., \& Luo, Y. M. (2006). Accumulation and distribution of polycyclic aromatic hydrocarbons in rice (Oryza sativa). Environmental Pollution, 140, 406-415. https://doi.org/10.1016/j.envpol.2005.08.004

USEPA. (1989). Risk assessment: guidance for superfund. In: Human Health Evaluation Manual (Part A), Interim Final, vol 1. Office of Emergency and Remedial Response, U.S. Environmental Protection Agency, Washington DC.

USEPA. (1999). Interim Report on Data and Methods for Assessment of 2,3,7,8-Tetrachlorodibenzo-p-dioxin Risks to Aquatic Life and Associated Wildlife. Office of Research and Development. EPA/600/R-93/055.

USEPA. (US Environmental Protection Agency). (2000). National Water Quality Inventory Report to the US Congress. EPA-841-R-02-001.

WHO (World Health Organisation. (2006). Safety Evaluation of Certain foods and Contaminants Prepared by the Sixty-second Meeting of WHO Expert Committee on Food. Series 72.

World Bank. (2014). Estimate for average life expectancy in Nigeria. World Bank Annual Report. Fiscal Year 2015 Regional Highlights. Retrieved August 28, 2016 from http://data.worldbank.org/country/nigeria

\section{Copyrights}

Copyright for this article is retained by the author(s), with first publication rights granted to the journal.

This is an open-access article distributed under the terms and conditions of the Creative Commons Attribution license (http://creativecommons.org/licenses/by/4.0/). 